\title{
Thermal mismatch dislocations produced by large particles in a strain-hardening matrix
}

\author{
David Dunand and Andreas Mortensen
}

Room 8-40I, Deparment of Materials Science and Engineering, Massachusetts Institute of Technology, Cambridge. MAOPIBO(U.S.A.)

\begin{abstract}
Silver chloride containing particles of irregular form of the order of $1 \mu \mathrm{m}$ in diameter is used as a model material for particle-reinforced metal matrix composites (MMCs). Matrix dislocations due to the mismatch of the coefficients of thermal expansion of the two phases are made visible to transmission optical microscopy through bulk decoration performed at room temperature. The plastic zone around the particles takes two forms, similar to what is observed around the glass microspheres: (a) rows of coaxial prismatic dislocation loops punched into the matrix and (b) a plastic zone of irregular form containing partially resolved tangled dislocations. A relationship between the inclusion volume and the volume of the plastic zone around the inclusion-developed for the geometrically tractable problem of a sphere embedded in a strain-hardening matrix deforming by slip-is found to be valid for particles of irregular form as well, which are more representative of the reinforcement used in MMCs than spheres are.
\end{abstract}

\section{Introduction}

Upon temperature change, stresses are generated in the vicinity of the reinforcement of a composite when the coefficients of thermal expansion (CTEs) of the two constituent phases are different. If these thermal stresses are high enough in metal matrix composites (MMCs), dislocations are produced at the interface and plastic flow occurs in the matrix. Plasticity due to thermal incompatibility has been studied experimentally in metals reinforced with particles, whiskers and fibers using etch pits [1,2], slip lines [3] as well as transmission electron microscopy (TEM) [4-10]. However, these techniques have shortcomings which preclude complete and accurate observation of the actual dislocation configurations in metals containing reinforcement of large size. One is that only material in the immediate vicinity of a free surface is examined, which perturbs both the dislocations and the stress state around the reinforcing phase. Also, while TEM is ideally suited to study dislocations around second phases of small dimensions, the size of the particles in most MMCs is much larger than the observable volume, in terms both of thickness and of width of the electron-transparent area. This restricts the observation to a small region of the plastic zone around large particles and precludes for example the investigation of long dislocations, or entire rows of punched dislocations extending far from the reinforcement.

To alleviate these shortcomings, we use silver chloride in the present investigation as a transparent model material for the matrix of particlereinforced MMCs. It is well known that silver chloride is very ductile (up to $400 \%$ tensile elongation) and that it exhibits dislocation mechanisms which are in most cases similar to those found in metals [11]. Individual dislocations can be decorated at room temperature within the transparent bulk of silver chloride (or bromide) by precipitation of metallic silver. They can then be resolved by high resolution transmission optical microscopy [12-14], provided that their density is not too high. Using plates of this material thin enough to keep light absorption and diffraction to acceptable levels (thickness up to millimeters), a volume as large as several square centimeters by $30 \mu \mathrm{m}$ can be decorated, making possible the observation of dislocations emitted 
by large particles found typically in MMCs in a manner that is impossible using TEM.

In what follows, we report direct experimental evidence of dislocations produced by thermal mismatch of irregular equiaxed glass particles in silver chloride. We then compare a simple geometrical model predicting the volume of the plastic zone in the case of spherical particles with present data on irregularly shaped particles.

\section{Experimental procedures}

In the fabrication of our samples, we closely followed the experimental procedures established by Mitchell and coworkers $[12,13,16]$. The matrix was prepared from $99.999 \%$ pure silver chloride (purchased from Engelhard, $\mathrm{OH}$, U.S.A.) doped with $0.05 \%$ cuprous chloride of the same purity (purchased from Strem Chemicals, MA, U.S.A.). The reinforcement used was borosilicate glass particles (glass BK10 purchased from MoSci Corporation, MO, U.S.A.) with a dimension range of $1-5 \mu \mathrm{m}$. Sample preparation, decoration and observation have been reported elsewhere [14].

\section{Results}

As in previous work [14], the samples exhibited extraneous precipitates that did not preclude the observation of the substructure: small black surface precipitates (1-5 $\mu \mathrm{m}$ in radius) after heat treatment and submicron random precipitation of silver (fog) within the volume upon exposure to actinic light. The matrix around the particles exhibited much higher den-
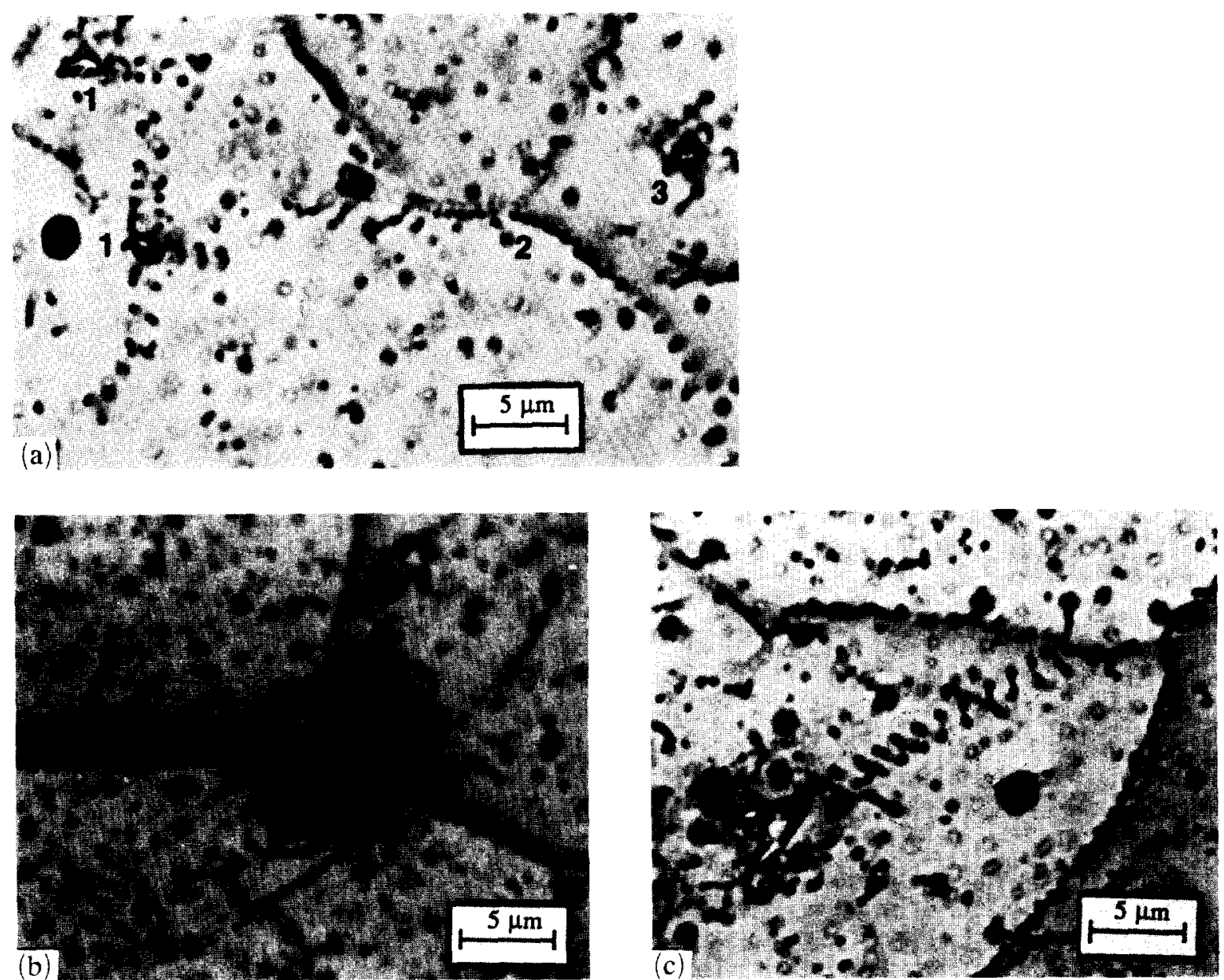

Fig. 1. (a) Decorated prismatic loops in $\mathrm{AgCl}$ punched by two glass particles (1). A wall of dislocations perpendicular to the image plane forms a subgrain boundary (2). Unresolved tangles are visible around another particle (3). (b) Partially resolved, decorated dislocations in $\mathrm{AgCl}$ forming a plastic zone around a glass particle located at the intersection of three grain or subgrain boundaries. (c) Glass particle in an $\mathrm{AgCl}$ matrix surrounded by an irregular zone of unresolved decorated dislocations and having emitted prismatic loops, some of which form a helix. The row of dislocations is blocked at a subgrain boundary. 
sities of decorated dislocations than the bulk of the matrix. In rare cases, the only resolvable dislocations were in the form of rows of prismatic loops punched along crystallographic directions (Fig. $1(a)$ ), a configuration found around microspheres as well [14]. The linear structure in Fig. 1(a) is a subgrain boundary consisting of dislocations perpendicular to the image plane. They can be resolved as points at the lower right corner of the micrograph and, by changing the focus of the microscope, can be followed in the crystal. Also visible in the same figure is a small particle surrounded by a dark decorated zone consisting of unresolved tangled dislocations. Another example of this plastic zone geometry is shown in Fig. 1 (b), where the dislocations in the tangles are partially resolved. This plastic zone morphology was by far the most common in the samples investigated. The plastic zone shape in most cases did not follow that of the corresponding particle. Spheres exhibited such regions of high dislocation densities as well, visible either as a regular spherical shell or as a zone of irregular form [14]. In several cases, both features described above were observed simultaneously around the same particle or sphere. Figure 1(c) illustrates such a mixed plastic zone morphology; the particle is surrounded by a decorated plastic zone of unresolved dislocation tangles and has also emitted a row of loops which extends at a large distance from the interface. The row is blocked by a subgrain boundary and the loops closest to the particle form a helix.

\section{Theory}

Various workers have used a continuum approach $[3,8,10,15,17-24]$ to predict the volume of plastic zones around inclusions embedded in metal. We use here an alternative model, which was proposed in a previous publication for spheres and long cylinders [14]. We solve the problem of a rigid mismatching sphere using a solution developed by Hill $[15$ ] for a growing hole which produces the same displacement at the sphere interface. This yields a solution identical with that of Kim et al. [10] developed following a different path:

$c_{s}=r_{\varsigma}\left(\frac{\Delta \alpha \Delta T E}{(1-v) \sigma_{v}}\right)^{1 / 3}$

where $\Delta \alpha$ is the difference between the CTEs of the two phases, $\Delta T$ is the temperature excursion, $E$ and $v$ are the matrix elastic modulus and Poisson's ratio, and $\sigma_{\mathrm{v}}$ is the matrix yield stress in tension. We now assume that the matrix in the plastic zone work hardens according to the classical relationship [25]:

$\sigma_{y}=2 \tau_{y 0}+2 A\left(s b \rho^{1 / 2}\right.$

where $\tau_{\mathrm{y}(0)}$ is the intrinsic flow stress of the matrix in shear, $G$ its shear modulus, $b$ the dislocations Burgers vector and the strain-hardening constant $A$ has a value of between 0.3 and 0.6 for metals [26] and of 0.4 for sodium chloride [27]. The dislocation density $o$ in the plastic zone around the particle is calculated from a simple model of dislocation punching and is assumed constant within the plastic zone. Using these simplifying assumptions, the radius $c_{\text {, }}$ of the plastic zone around a sphere of radius $r_{s}$ is given by

$$
\begin{aligned}
& \left(\frac{c_{s}}{r_{s}}\right)^{\prime \prime}-\left(1+C_{1}+2 C_{2}\right)\left(\frac{c_{s}}{r_{s}}\right)^{\prime \prime} \\
& +\left(C_{2}^{2}+2 C_{2}\right)\left(\frac{c_{s}}{r_{s}}\right)^{3}-C_{2}^{2}=0
\end{aligned}
$$

where $C_{1}$ and $C_{2}$ are dimensionless constants:

$$
\begin{aligned}
& C_{1}=\frac{6 \sqrt{2} \Delta \alpha \Delta T b}{r_{5}}\left(\frac{A G}{\tau_{y 1}}\right)^{2} \\
& C_{2}=\frac{\Delta \alpha \Delta T E}{2(1-v) \tau_{\mathrm{y} 11}}
\end{aligned}
$$

To the best of our knowledge, all models available in the literature (and the model proposed above is no exception) use simple idealized shapes such as cylinders, spheres or ellipsoids to describe particles in MMCs. However, commercially available particulate reinforcements used in most MMCs usually exhibit an irregular form and sharp angles. To model such a system would necessitate a complex statistical treatment as well as a thorough knowledge of the geometrical parameters best describing the particles; we make the simplifying assumption that a particle of irregular shape and a sphere with the same volume both generate a plastic zone of same volume. This is reasonable if the sizes of the emitted loops are about the same in both cases, since the volume to be displaced from the interface by dislocations is dictated by the total thermal mismatch, which is itself independent of the 
particle shape. We define the equivalent radius $r_{\mathrm{e}}$ of the particle as that of a sphere having the same volume, and the equivalent radius $c_{\mathrm{e}}$ of the plastic zone around the particle as that of the spherical plastic zone around the sphere.

While the volume of a sphere can be very simply determined from the surface of its projection, the same is not true for bodies of irregular form. Certain general topological relationships hold, however, for a system of convex bodies of single shape and size [28]. The volume $V$ of a body is a function of its mean projected area $\overline{A^{\prime}}$ and its mean intercept length $\overline{L_{3}}$;

$$
V=\overline{A^{\prime} L_{3}}
$$

The mean projected area can in turn be expressed in terms of the mean intercept length of the projected area $\overline{L_{2}^{\prime}}$ and the mean projected height of the body $\overline{H^{\prime}}$ :

$\overline{A^{\prime}}=\overline{L_{2}^{\prime} H^{\prime}}$

The mean intercept length $\bar{L}_{3}$ is a three-dimensional property which cannot be expressed in terms of two-dimensional projected values only. This reflects the fact that information is lost in the passage from a three-dimensional object to a twodimensional projection, unless the shape of the object is known independently. We define a constant $K$ such that $K=\overline{L_{3} / L_{2}^{\prime}}$. This shapedependent constant was calculated for different regular body shapes, starting from the values of $V, \overline{A^{\prime}}$ and $\overline{H^{\prime}}$ given by Underwood [29], and found to vary within a relatively narrow range, extending from 0.50 to 0.88 . Equations (5) and (6) can then be combined into an expression for the volume which contains only projected variables $\overline{A^{\prime}}$ and $\overline{H^{\prime}}$ and the parameter $K$. Since the particles are randomly oriented in the matrix, the area projected in the image plane of the microscope is taken as the mean projected area of the particle and the mean projected height is approximated as the mean projected height of its projected area. Both above assumptions are valid if a large number of particles are considered. Since the shape of the particle is not known, the average of the minimum and maximum values for $K$ given above is taken $(K=0.69)$.

\section{Discussion}

Prismatic punching in metals containing submicroscopic particles (see for example refs. 30 and 31) as well as in silver halide containing microspheres $[14,32]$ and fibers $[14,33]$ has been reported by many investigators. Figure 1 (a) confirms that this mechanism is operative for particles with volumes from about $1 \mu \mathrm{m}^{3}$ to a few hundred cubic microns. The scarcity of particles which exhibited trains of loops as their only plastic zone suggests that such prismatic loops easily become entangled after they have been emitted, thus forming the plastic zone of high dislocation density found around particles (Fig. 1(b)) and microspheres [14]. TEM investigations of submicroscopic particles in metals $[34,35]$ have shown that such entanglement is common around smaller particles. The hypothesis that the tangled dislocations in the plastic zone were originally prismatic loops is confirmed by the frequent observation of particles and microspheres showing one or more rows of loops in addition to a shell of dense dislocations (Fig. 1(c)). Such a configuration will form if relaxation begins with nucleation and emission of prismatic loops on non-adjacent glide cylinders, followed by the activation of additional glide cylinders overlapping with the original glide cylinders and subsequent entanglement, similar to what has been observed with smaller particles in metals [36]. An observation reinforcing the above hypothesis is that no particle was found with adjacent rows of loops (i.e. with rows forming an angle of $\pi / 3$ ). Quite often, however, two rows were visible outside the shell of tangled dislocations, almost invariably at opposite sides of the particle. These observations support the choice of loop geometry made above for the calculation of dislocation density and plastic zone volume, as with spheres [14]. A feature specific to particles observed in many other instances is the emission of a row of loops from sharp corners of the particles (Fig. 1(c)). As with short fibers [14], the ends of which had punched rows of prismatic loops, this can be explained by the stress concentration effect of cuspidal points which must help nucleation of the loops.

The projected area of the particles and their associated plastic zone were cut and weighed from enlarged micrographs; the mean projected length was measured directly on the enlargements. The relative error for each of these measurements is estimated at $5 \%$ for the smallest particles and less for the larger particles. Taking into account the maximum error in the constant $K$ estimated above as $28 \%$, the maximum relative error in the calculation of the volume using the 


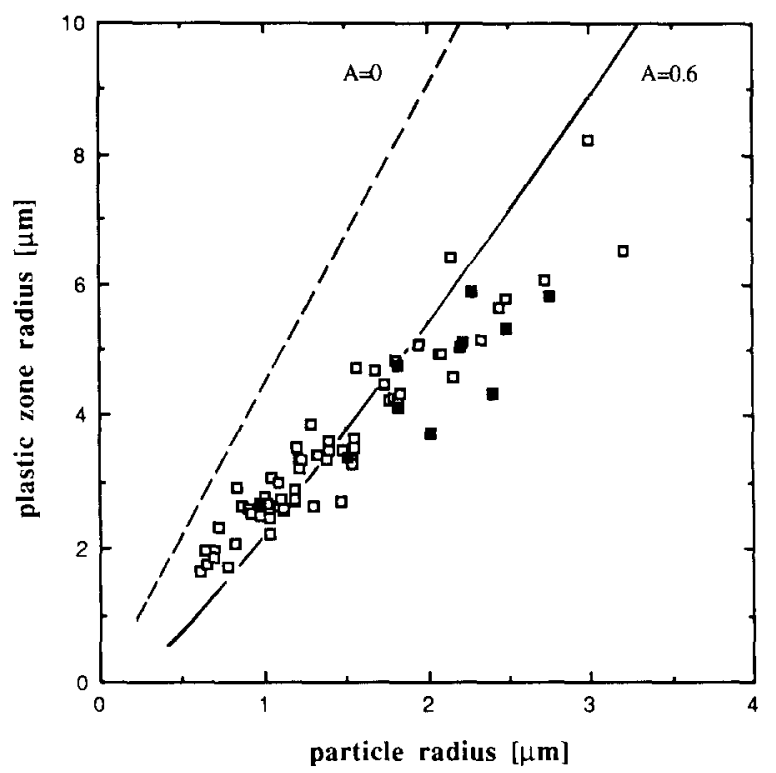

Fig. 2. Equivalent radius of the plastic zone surrounding particles in an $\mathrm{AgCl}$ matrix as a function of the equivalent radius of the corresponding particle: - plastic zone formed of tangled dislocations and row of loops (see Fig. 1(c)): $\square$, plastic zone formed of tangled dislocations only isee Fig. 1(b): - - strain-hardening matrix with $A=0.6$ (eqn. (3i): --- , non-strain-hardening matrix (eqn. $(1)$ ).

procedure outlined above is therefore about $43 \%$ for the small particles and less for the large particles. The maximum error in the equivalent sphere radius is thus on the order of $15 \%$. The only particles considered were those which had a clearly defined plastic zone and which were far enough from neighbouring particles so that their plastic zones were not in contact.

The equivalent radii of the particles and their plastic zones are plotted against each other in Fig. 2. As expected, particles which had punched loops before tangles were formed (Fig. 1(c)) tended to display a smaller plastic zone (full squares in Fig. 2). Also plotted in Fig. 2 is the solution of eqn. (3), using a value of $5.8 \times 10^{-6}$ $\mathrm{K}^{-1}$ for the CTE of the glass [37], physical constants for $\mathrm{AgCl}[14]$ and values of $\Delta T=100 \mathrm{~K}$ and $A=0.6$ previously determined with spheres and fibers under the same conditions [14]. Observation and theory match very well over the whole range of volumes, which spans more than two orders of magnitude. Equation (1) which neglects the strain hardening of the matrix overestimates the plastic zone volume (broken line in Fig. 2). The good match between data and theory also proves that the assumption-made above-that irregularly shaped particles, as they occur in most
MMCs, can be treated as equivalent spherical particles of the same volume is legitimate. While the shape of the plastic zone is also irregular and cannot be predicted, its volume is very well described by the volume of a spherical plastic zone resulting around a sphere of identical volume in a workhardening matrix. However, the plastic zone does not form a shell of constant thickness around the particle, which might be because relaxation starts earlier at some places than at others or because entanglement does not occur at the same distance from the interface. Large errors may therefore be introduced if TEM measurements (such as dislocation density or plastic zone extent) are made only at one limited region of the interface and are generalized to the whole plastic zone.

\section{Conclusions}

(1) Silver chloride containing irregularly shaped glass particles is used as a model material for the study of thermally induced dislocations in MMCs. The dislocations are observed by transmission optical microscopy after having been decorated in the bulk matrix at room temperature. Despite the lower resolution achievable, this decoration technique complements investigations of MMCs by electron microscopy, because a large volume of undisturbed material can be observed.

(2) To relieve stresses due to the mismatch in CTEs with the matrix, particles generate (i) rows of prismatic loops or (ii) tangles of dislocations, forming a well-defined surrounding plastic zone. In certain cases, these two features coexist. The shape of the plastic zone does not correlate with that of the particle. These phenomena are also observed around microspheres of similar size as the particles [14]. An additional feature specific to the particles is the nucleation of loops at sharp corners, probably stimulated by the local stress concentration.

(3) A model that was developed and confirmed for a low volume fraction of microspheres in a strain-hardening matrix [14] is adapted to particles of irregular shape. Their plastic zone volumes, estimated from stereological relationships, are found to match well with the prediction of the model. Particles of irregular form can thus be assimilated to spheres, thereby significantly simplifying the modelling of the plastic zone volume in particulate MMCs. 


\section{Acknowledgments}

This work was supported by the Office of Naval Research under Contract N00014-89-J1541 , monitored by Dr. S. G. Fishman.

We gratefully acknowledge advice in the initial stages of our experimental research by Professor J. W. Mitchell of the University of Virginia.

\section{References}

1 K. K. Chawla and M. Metzger, J. Mater. Sci., 7(1972) 34.

2 K. K. Chawla and M. Metzger, Metall. Trans. A, 8(1977) 1680 .

3 Y. Flom and R. J. Arsenault, Mater. Sci. Eng., 75 (1985) 151.

4 R. J. Arsenault and R. M. Fisher, Scr. Metall., 17 (1983) 67.

5 S. P. Rawal, L. F. Allard and M. S. Misra, in A. K. Dhingra and S. G. Fishman (eds.), Interfaces in Metal Matrix Composites, AIME, New York, 1986, p. 211.

6 S. P. Rawal, L. F. Allard and M. S. Misra, in F. L. Matthews, N. C. R. Buskell and J. M. Hodginson (eds.), Proc. 6th Int. Conf. on Composite Materials, Elsevier Applied Science, Barking, Essex, 1987, p. 2.16.

7 M. Vogelsang, R. J. Arsenault and R. M. Fisher, Metall. Trans. $A$, 17(1986) 379 .

8 M. Taya and T. Mori, Acta Metall., 35(1987) 155.

9 Q. Li, J. Megusar, L. J. Masur and J. A. Cornie, Mater. Sci. Eng. A. $117(1989) 199$.

10 C. T. Kim, J. K. Lee and M. R. Plichta, Metall. Trans. A, $21(1990) 673$

11 M. T. Sprackling, The Plastic Deformation of Simple Ionic Crystals, Academic Press, New York, 1976.

12 P. V. Clark and J. W. Mitchell, J. Photogr. Sci. 4 (1956) 1.

13 J. W. Mitchell, Dislocations and Mechanical Properties of Crystals, Wiley, New York, 1957, p. 69.

14 D. C. Dunand and A. Mortensen, Acta Metall. Mater., in the press.
15 R. Hill, The Mathematical Theory of Plasticity, Oxford University Press, Oxford, 1950.

16 J. M. Hedges and J. W. Mitchell, Philos. Mag., 7 (1953) 357.

17 A. R. T. de Silva and G. A. Chadwick, J. Mech. Phys Solids, 17(1969) 387

18 C. A. Hoffman, J. Eng. Mater. Technol., $95(1973) 55$.

19 G. Garmong, Metall. Trans., 5(1974) 2183.

20 J. K. Lee, Y. Y. Earmme, H. I. Aaronson and K. C. Russell, Metall. Trans. A, 11 (1980) 1837.

21 Y. Y. Earmme, W. C. Johnson and J. K. Lee, Metall. Trans. $A, 12$ (1981) 1521.

22 M. Vedula, R. N. Pangborn and R. A. Queeney, Composites, $19(1988) 55$.

23 M. Vedula, R. N. Pangborn and R. A. Queeney, Composites, 19(1988) 133.

24 I. Dutta, D. L. Bourell and D. Latimer, J. Compos. Mater, $22(1988) 829$.

25 G. I. Taylor, Proc. R. Soc. London, Ser. A, 145 (1934) 362 .

26 H. Wiedersich, J. Met., 16(1964) 427.

27 W. inder Schmitten and P. Haasen, J. Appl. Phys., 32 (1961) 1790 .

28 E. E. Underwood, J. Microsc., 95(1972) 25.

29 E. E. Underwood, Quantitative Stereology, AddisonWesley, Reading, MA, 1970, p. 90.

30 A. Lawley and H. L. Gaigher, Philos. Mag., 8 (1963) 1713.

31 R. W. K. Honeycombe, The Plastic Deformation of Metals, American Society for Metals, Metals Park, OH, 2nd edn., 1984, p. 197.

32 J. W. Mitchell, Growth and Perfection of Crystals, Wiley, New York, 1958, p. 386.

33 L. M. Childs and C. B. Slifkin, Br. J. Appl. Phys., 16 (1965) 771.

34 M. H. Lewis and J. M. Martin, Acta Metall., 11 (1963) 1207.

35 M. F. Ashby, S. H. Gelles and L. E. Tanner, Philos. Mag., 19(1969) 757.

36 B. J. Makenas and H. K. Birnbaum, Acta Metall., 28 (1980) 979.

37 Ceramic Sources, American Ceramic Society, Columbus, $\mathrm{OH}, 1988$, p. 234. 